\title{
Inmigración, estrategias de aculturación y valores laborales: un estudio exploratorio
}

\section{Inmigration, acculturation strategies and work values: an exploratory study}

\begin{tabular}{c} 
ALVARO RETORTILLO OSUNA ${ }^{1}$ \\
HENAR RODRÍGUEZ NAVARRO ${ }^{2}$ \\
\hline Fecha de Recención: $04-11-2007$
\end{tabular}

\section{RESUMEN}

En las últimas dos décadas, España se ha convertido en uno de los principales países de destino de la inmigración internacional, con las consecuencias que ello conlleva a nivel económico, laboral, cultural etc., es decir, sus implicaciones sociales son muchas y de muy variada índole. Dentro de la complejidad y multidimensionalidad inherente al fenómeno, el presente artículo muestra un resumen de investigación que pretende poner en conexión las estrategias de aculturación puestas en práctica por las personas inmigrantes, relacionándolas con uno de los aspectos clave que influyen en su adaptación laboral y el significado que atribuyen al trabajo remunerado: los valores laborales. Desde un prisma exploratorio, nuestro estudio muestra la asociación entre los valores laborales de una muestra de inmigrantes en función de sus estrategias de aculturación, derivadas de la aplicación del Modelo de Berry, con el fin de detectar a qué aspectos del trabajo otorgan más importancia y cómo influyen las relaciones con la cultura de origen y de acogida en esa atribución de valor.

\section{ABSTRACT}

Over the last two decades, Spain has become a major immigration country with the associated economical, labour, cultural impact ant its implications. This paper departs from a

${ }^{1}$ Facultad de ciencias del Trabajo.alvareto@psi.uva.es

2 Facultad de Educación y Trabajo Social.henarod@psi.uva.es 
Inmigración, estrategias de aculturación y valores laborales: un estudio exploratorio

consideration of the complexity and multi-dimensionality of this phenomenon. A review is made of literature regarding the connexion between acculturation strategies deployed by the immigrants and social values -a key component of work fit and work meaning. Acculturation strategies in this exploratory study are based on Berry's Model. These strategies identify which work aspects are awarded more importance and to what extent cultures from both the country of origin and the host country influence the attribution of value.

\section{PALABRAS ClAVE}

Inmigración, Estrategias de aculturación, Valores laborales, Inclusión social,

\section{KEY WORDS}

Immigration, Acculturation strategies, Work values, Social inclusion. 
Una de las principales transformaciones que ha experimentado la sociedad española en las últimas décadas y que más peso específico ha tenido en el cambio de su estructura social ha sido la llegada de inmigrantes a gran escala. Se trata de un proceso que se venía dando en otros países de Europa desde hace décadas, pero que en España es relativamente reciente y la configura actualmente como un foco de atracción de la inmigración internacional, situándose a la par de otros países europeos con mucha mayor tradición migratoria como Francia o Alemania, con las consecuencias e implicaciones sociales que el fenómeno tiene a todos los niveles (social, económico, político, cultural, etc.).

Dado que actualmente la inmigración es un fenómeno estructural de las sociedades desarrolladas y que tiene una clara voluntad de permanencia, es inevitable que dos o más comunidades culturalmente diferenciadas entren en contacto y se produzcan cambios en muchos aspectos de la vida diaria de sus miembros. Por tanto, se genera un proceso de aculturación que afecta tanto a los nuevos inquilinos como a la comunidad receptora. La aculturación hace referencia a la aprehensión de una determinada cultura, supone el conocimiento, la interiozación, valoración, identificación y manejo dinámico de los valores culturales propios y de las demás culturas en contacto. A pesar de que la aculturación se ha tratado ampliamente en disciplinas como la Antropología, nos centraremos en la aculturación psicológica, que puede ser definida como el proceso mediante el cual las personas cambian, siendo influidas por el contacto con otra cultura y participando en los cambios generales de su propia cultura (Berry, 1990). El proceso de aculturación se da tanto en inmigrantes como en autóctonos, aunque normalmente existe una cultura (la dominante) que ejerce su influencia sobre la otra (la subordinada). Las numerosas investigaciones llevadas a cabo por Berry y sus colaboradores en el campo de la Psicología Transcultural dieron lugar a la formulación de un Modelo de Aculturación (Berry, Kim, Power, Young y Bujaki, 1989; Berry, 1990). Este modelo y sus posteriores variaciones y/o adaptaciones (Berry, 2005, 2006a) ha sido una de las bases de la investigación teórica y empírica sobre la materia. Desde el punto de vista de los grupos no dominantes, el modelo se fundamenta en las estrategias que adoptan los inmigrantes para enfrentarse a dos decisiones cruciales en sus vidas: 1) decidir si se mantiene su propia cultura en la sociedad de acogida y en qué grado, además de las relaciones con las personas de su mismo origen; y 2) decidir su nivel de participación en la nueva sociedad, si se relacionan o no con los autóctonos y la naturaleza de esa relación. La combinación de estos dos elementos da lugar a cuatro posibles estrategias de aculturación a adoptar por los individuos: integración, asimilación, separación y marginación. Si existe un deseo o posibilidad de articular la práctica de las propias culturas de origen y a su vez participar activamente en la sociedad de acogida, nos encontraremos ante la opción "integración". Si los individuos no quieren o deciden no mantener su identidad cultural y buscar una interacción diaria con otras culturas, estaremos ante la opción "asimilación", donde las personas prefieren apartar su cultura originaria y ser absorbidos por la de la sociedad de destino. En contraste con lo anterior, cuando los inmigrantes valoran sobre todo su cultura y rechazan el contacto con la cultura de acogida, nos encontraremos ante la opción "separación". Por último, cuando el interés o la posibilidad 
Inmigración, estrategias de aculturación y valores laborales: un estudio exploratorio

de mantener la cultura de origen y participar en la sociedad de acogida es pequeña, estaremos ante la opción “marginación”.

En el presente artículo nos centraremos en la relación existente entre las dimensiones de la aculturación y el significado que tiene para las personas inmigrantes una de las actividades humanas que más influencia tiene sobre la vida del ser humano: el trabajo. La sociedad contemporánea se ha transformado en una sociedad laboral (Claes, 1987; Ovejero, 2006; Alonso, 2007), donde la necesidad de trabajar se ha convertido en el deseo de trabajar. El empleo remunerado no sólo desempeña un papel económico para las personas, sino que cumple una importante serie de funciones psicosociales tales como la autorrealización del sujeto, proporciona prestigio y estatus social, es una fuente de identidad personal etc. (Salanova, Prieto y Peiró, 1993; Ovejero, 2006). Según el proyecto MOW (1987), el significado del trabajo es el conjunto de valores, creencias y expectativas que el individuo tiene sobre el mismo, considerándolo como un constructo psicológico o individual, un resultado de la estructura cognitiva que es el resultado de la experiencia del sujeto y del ambiente durante el proceso de socialización laboral.

Según Rockeach (1973), un valor es una creencia constante acerca de un determinado modo de comportamiento o un estado vital definitivo que se contrapone a otro u otros que se consideran opuestos o no compatibles. Tales creencias se organizan de forma permanente para configurar modos deseables de conducta o estados de existencia definitivos durante un periodo de tiempo prolongado de relativa importancia. Los valores son aprendidos y varían en cuanto a su identidad y contenido en función de los sujetos, el contexto y los grupos sociales. Por tanto, los valores condicionan las conductas y las reacciones emocionales de los individuos, ya que operan como normas que van más allá de los objetos y las situaciones concretas. Cuando hablamos de valores laborales estamos refiriéndonos a aquello que las personas esperan de su trabajo, por lo que tal propiedad es usada en los juicios de valor sobre aquellos aspectos deseables del empleo. Por otra parte, los valores tienen un equilibrio, un punto óptimo que varía en función de la cantidad poseída y deseada de cada uno (MOW, 1987). Como consecuencia de todo ello, los valores laborales reflejan las preferencias de cada individuo, son una necesidad activada.

Herzberg (1959), con su Teoría de los Dos Factores, postuló la existencia de dos grupos o clases de aspectos laborales: factores extrínsecos y factores intrínsecos. Los primeros están referidos a las condiciones de trabajo en el sentido más amplio, tales como el salario, las políticas de empresa, el entorno físico, la seguridad en el trabajo, etc. Los factores intrínsecos serían aquellos que son consustanciales al trabajo: contenido del mismo, responsabilidad, logro, etc. Los valores laborales asociados a los dos factores propuestos hacen que éstos se subdividan a su vez en valores laborales intrínsecos y extrínsecos. $\mathrm{Su}$ distribución jerárquica hace que se les otorgue más importancia a unos que a otros (MOW, 1987; Ravlin y Megglino, 1987). En conexión con esta idea y con la clásica jerarquía de necesidades de Maslow (1954), numerosos estudios han demostrado la prevalencia de los valores extrínsecos sobre los intrínsecos (Mortimer, Lorente y Kunda, 1986; Topalova y Ruiz Quintanilla, 1992), ya que son los que permiten que las necesidades básicas 
de la persona queden cubiertas. No obstante, existen revisiones de este concepto que niegan incluso la existencia de una jerarquía de valores (Wabha y Bridwell, 1976). Cabe destacar en este punto la distinción entre valores materialistas y postmaterialistas hecha por Inglehart (1991, 1997) e Inglehart y Welzel (2006). Según esta tesis, los valores de las sociedades occidentales han ido evolucionando desde la atribución de una importancia suprema al bienestar material y a la seguridad física hacia un énfasis mucho mayor en la calidad de vida, con lo cual la autorrealización personal (y por ende los valores intrínsecos del trabajo) cobran una relevancia que antes no poseían.

El objetivo de nuestro estudio no es otro que el de poner en relación el proceso de aculturación (y, más concretamente, el modelo propuesto por Berry y sus colaboradores) y los valores laborales del grupo "no dominante", es decir, de los inmigrantes. Las personas responden de mantera diferente de acuerdo a sus valores y expectativas principales (Lawler, 1973; Stitt-Gohdes, 1997; Taris, 2003) por lo que, de acuerdo con las teorías dominantes sobre el desarrollo de carrera, las incongruencias entre los valores laborales y las recompensas derivadas del trabajo tienen consecuencias negativas sobre la satisfacción laboral, los intentos de abandono del puesto de trabajo etc., es decir, sobre la adaptación laboral de la persona (Locke, 1976; Holland, 1976; Shields y Ward, 2001; Ovejero, 2006).

Son varios los estudios realizados que tratan de evaluar los valores laborales en distintos colectivos insertos en el mercado laboral o en disposición de hacerlo. Tras la relativamente reciente conversión de España en un país de inmigración y la creación de una nueva categoría social, el inmigrante (Cachón, 2003), que en múltiples ocasiones se configura como un trabajador invisible (Martínez Veiga, 2004) y tras la incertidumbre asociada al proceso de globalización en el cual se encuentra encuadrada hoy en día la humanidad (Falk, 2002; Ovejero 2004) consideramos conveniente realizar un ejercicio de aproximación al conocimiento de los valores laborales que tienen las estas personas dentro del proceso de aculturación que experimentan como consecuencia del asentamiento en la nueva sociedad, ya que tendrá fuertes implicaciones en el ajuste laboral de las mismas y, por extensión, en su inclusión social. El proceso de aculturación no afecta exclusivamente a los nuevos inquilinos, sino que se articula como un proceso bidireccional que tiene implicaciones para el conjunto de la sociedad (Berry, 2005), por lo que los efectos de la adaptación en el trabajo de las personas inmigrantes se extienden también de manera directa o indirecta sobre los autóctonos y sobre la estructura social. Para realizar el acercamiento a la comprensión de los valores laborales de la población inmigrante hemos enmarcado la investigación en el seno de las relaciones con la cultura propia y la autóctona, así como con los miembros del grupo dominante y no dominante (es decir, en el modelo de aculturación propuesto por Berry), ya que el contexto laboral está condicionado, inevitablemente, por el proceso de aculturación, una transición que supone que la persona migrante experimente cambios de rol y/o de escenario y que implica la necesidad de reajustar varias dimensiones de la vida diaria las como la dimensión familiar, social, laboral, educativa, cultural etc. (Bronfenbrenner, 1987; Searle y Ward, 1990; Ward y Kennedy, 1994; Navas et al, 2004; Berry, 2005, 2006a). 
Inmigración, estrategias de aculturación y valores laborales: un estudio exploratorio

\section{MÉTODO}

\section{Participantes}

La muestra fue seleccionada mediante muestreo por cuotas, estratificado por zona de procedencia, edad y estatus de actividad laboral, con el objetivo de obtener una visión relativamente amplia y representativa de los colectivos inmigrantes residentes en la provincia. La muestra final estuvo formada por 160 personas inmigrantes, residentes y empleados en la provincia de Valladolid. La edad media de las personas encuestadas es de 34,68 años, con una desviación típica de 8,46. En cuanto al sexo, el porcentaje de hombres es del $56,25 \%$ por un $43,75 \%$ de mujeres. En lo relativo a la procedencia, los sujetos seleccionados pertenecen a los tres colectivos más numerosos del ámbito territorial objetivo de estudio: latinoamericanos $(\mathrm{N}=54)$, europeos del este $(\mathrm{N}=53)$ y magrebíes $(n=53)$. Asimismo, con el objetivo de conseguir una mayor diversidad de la muestra, se incluyeron personas con distintos niveles educativos (analfabetos, estudios primarios, estudios secundarios, formación profesional u ocupacional, estudios universitarios y otra formación superior). En cuanto a la cualificación exigida para el desempeño del puesto de trabajo, un $23,75 \%$ posee un empleo cualificado, frente a un $76,25 \%$ que realiza tareas que no requieren una cualificación concreta.

\section{Instrumentos}

Para la medición de las estrategias de aculturación, es decir, las relaciones con la cultura propia y con la del país de acogida, se ha tomado como base la Escala de Estrategias de Aculturación de Kosic (1998), que a su vez se deriva parcialmente de dos escalas anteriores de Dona y
Berry (1994) y Ward y Kennedy (1993). Por una parte, la escala muestra el grado en el que los inmigrantes se sienten parte de la cultura de acogida y la interacción con sus miembros y, por otra, la identificación con el exogrupo autóctono y sus formas culturales. La escala incluye ítems que se refieren a las relaciones sociales (cantidad y calidad de relaciones de amistad con los miembros de la sociedad de acogida y las personas de su mismo origen), dificultades sociales (relaciones con los empleadores, con los autóctonos con los que la persona se interrelaciona en la vida diaria etc.), la nostalgia que el sujeto tiene de la propia cultura (comida, amigos, música, televisión etc.) y la adaptación a la cultura autóctona (comida, idioma etc.). La escala contiene 29 ítems con un modelo de escala Likert de 5 categorías, donde las puntuaciones más cercanas a 5 indican una relación fuerte y las más cercanas a 1 una relación débil. El análisis de factorial revela dos factores similares a los encontrados por Kosic: a) un primer factor, haciendo referencia al mantenimiento de la cultura de origen y a las relaciones con las personas procedentes de su misma zona, que contiene 11 ítems que explican la tendencia hacia la nostalgia de su cultura y país y; b) un segundo factor, relativo a la participación en la sociedad de acogida y a las relaciones con los autóctonos, que aglutina 18 ítems que se refieren al grado en que se sienten aceptados por los españoles, el sentido de sus relaciones con ellos y la participación en la sociedad de acogida.

Los valores laborales fueron medidos a través de la escala desarrolada por el WOSY (Work Socialization of Youth) International Research Group (1989b). Dicha escala evalúa la actitud de los trabajadores hacia los valores laborales extrín- 
secos (salario, condiciones de trabajo, seguridad laboral, oportunidades de promoción y jornada) e intrínsecos (oportunidades de aprendizaje, autonomía, variedad, trabajo interesante y ajuste de habilidades) del trabajo. Las puntuaciones oscilan entre 1 y 5 puntos, siendo las más cercanas a 5 las que expresan mayores valores laborales. El análisis factorial revela la existencia de dos factores que se corresponden con las dos subescalas presentes en el instrumento: valores extrínsecos (5 ítems) e intrínsecos (6 ítems).

Se comprobaron las adecuadas propiedades psicométricas de ambas escalas (con el programa SPSS 11.5) mediante: a) el análisis de la fiabilidad, obteniendo, en el caso de la Escala de Estrategias de Aculturación unos coeficientes alfas de $.96, .92$ y .94 , para las dos subescalas y la escala global respectivamente y $.94, .73$ y .87 en el caso de la escala que mide los valores laborales; y b) la normalidad del modelo estadístico, homoscedasticidad y no colinealidad, revelando tanto el histograma de residuos como el gráfico de probabilidad la existencia de normalidad, el diagrama de dispersión de los residuos tipificados nos confirmó la igualdad de varianzas y los índices de tolerancia e inflación nos hicieron descartar la existencia de colinealidad entre las variables independientes.

\section{Procedimiento}

Previamente a la aplicación de la encuesta se realizó un estudio piloto con 25 sujetos cuyos resultados fueron satisfactorios, aunque se tuvo que reformular el enunciado de determinados ítems para facilitar su comprensión, sobre todo como consecuencia de la existencia de otros idiomas maternos distintos al castellano entre los sujetos participantes. El acceso a

Tabla 1. Análisis factorial: escala de valores laborales

\begin{tabular}{|l|c|c|}
\hline & \multicolumn{2}{|c|}{ Factor } \\
\hline & 1 & 2 \\
\hline Muchas oportunidades de aprender cosas nuevas & .909 & \\
Buenas relaciones con los compañeros & .770 & \\
Buenas oportunidades para ascender en el trabajo & & .569 \\
Buen horario de trabajo & & .731 \\
Un trabajo variado & .902 & \\
Un trabajo interesante & .881 & \\
Buena estabilidad en el trabajo & & .594 \\
Buen ajuste entre tus habilidades y experiencias y las que exige el & .903 & \\
trabajo & & .733 \\
Un buen salario & & .723 \\
Buenas condiciones físicas & .875 & \\
Mucha autonomía & $45.448 \%$ & $19.717 \%$ \\
\hline Varianza explicada (porcentaje) & $19.717 \%$ & $65.165 \%$ \\
\hline Varianza explicada (porcentaje acumulado) & & \\
\hline
\end{tabular}


Inmigración, estrategias de aculturación y valores laborales: un estudio exploratorio

la muestra fue facilitado por la colaboración de distintas organizaciones no gubernamentales (fundamentalmente asociaciones de ayuda al inmigrante) que tratan con el colectivo objeto de estudio. Dichas asociaciones participaron desinteresadamente en la investigación, facilitando el contacto con inmigrantes que reunían el perfil requerido, cediendo espacios para la aplicación de los cuestionarios y traduciendo los mismos en aquellos casos en los que el idioma suponía una barrera para la comprensión de los ítems. La aplicación del instrumento fue realizada por los investigadores del proyecto en la gran mayoría de los casos y por colaboradores preparados a tal efecto en el resto. Para tratar de mitigar el posible efecto perturbador de la deseabilidad social, se informó adecuadamente a los evaluados sobre la existencia de detectores de distorsión y se les informó de que aquellos sujetos que denotasen deseabilidad social y otros sesgos serían excluidos de la investigación (Salgado, 2005).

\section{Hipótesis del estudio}

En coherencia con la literatura revisada, para el presente estudio partimos de las siguientes hipótesis:

a) De acuerdo con la teoría de la jerarquía de necesidades, es de esperar que los valores laborales extrínsecos superen en importancia atribuida a los intrínsecos, ya que son aquellos que implican la satisfacción de las necesidades más básicas de la persona Por tanto, se vislumbra así una distribución jerárquica de los valores laborales, desechando aquellas concepciones que cuestionan la propia existencia de tal jerarquía. En coherencia con esta idea, los valores laborales extrínsecos serán pree- minentes en todos los grupos de aculturación.

b) Como consecuencia de la evolución de las sociedades occidentales hacia una creciente preferencia por los valores postmaterialistas (lo que conlleva un aumento de la importancia atribuida a los valores intrínsecos del trabajo), aquellas personas incluidas dentro de los grupos de integración y asimilación, es decir, aquellas que tienen una relación fuerte con la cultura de acogida, poseerán un mayor número de valores laborales intrínsecos que aquellos que se encuadran dentro de los grupos de separación y marginación.

\section{RESULTADOS}

En primer lugar, se analizaron los resultados generales sin tener en cuenta la actitud de aculturación de los sujetos participantes en la investigación. La puntuación promedio de la escala de valores intrínsecos es de 3.90, mientras que la de valores extrínsecos es de 4.33. La escala de valores globales consigue una puntuación de 4.09. Dentro de los valores laborales intrínsecos, los que mayor puntación obtienen son las relaciones con los compañeros $(\mathrm{M}=4.22)$ y el deseo de que el trabajo sea interesante $(\mathrm{M}=4.14)$. En el caso de los valores extrínsecos, destacan el salario $(\mathrm{M}=4.61)$ y la estabilidad en el empleo $(\mathrm{M}=4.51)$.

En segundo lugar, se efectuaron una serie de pruebas para determinar si la zona de procedencia del inmigrante tenía influencia sobra las dimensiones de la aculturación y los valores laborales. Las pruebas ANOVA y post-hoc de Scheffé revelan que el origen de los sujetos no influye en las relaciones con la cultura de 
Tabla 2. Puntuaciones medias de los valores laborales intrínsecos y extrínsecos

\begin{tabular}{|c|c|c|}
\hline & Media & Desv.Típica \\
\hline \multicolumn{3}{|l|}{ Valores intrínsecos } \\
\hline Autonomía & 3.88 & 1.10 \\
\hline Relaciones con los compañeros & 4.22 & 0.96 \\
\hline Trabajo variado & 3.71 & 1.07 \\
\hline Trabajo interesante & 4.14 & 0.90 \\
\hline Ajuste entre habilidades y conocimientos & 3.72 & 1.08 \\
\hline Aprender & 3.70 & 1.05 \\
\hline Total valores intrínsecos & 3.90 & 0.91 \\
\hline \multicolumn{3}{|l|}{ Valores extrínsecos } \\
\hline Promoción & 4.12 & 0.74 \\
\hline I Iorario & 4.21 & 0.68 \\
\hline Estabilidad & 4.51 & 0.61 \\
\hline Salario & 4.61 & 0.55 \\
\hline Condiciones lísicas & 4.18 & 0.78 \\
\hline Total valores extrínsecos & 4.33 & 0.46 \\
\hline Total valores laborales & 4.09 & 0.58 \\
\hline
\end{tabular}

Tabla 3. Correlaciones entre las dimensiones de la aculturación y los valores laborales

\begin{tabular}{|c|c|c|}
\hline & Valores laborales intrínsecos & Valores laborales extrínsecos \\
\hline Relaciones cultura de origen & .241 & .047 \\
\hline Relaciones cultura de acogida & .780 & .208
\end{tabular}

origen $\mathrm{F}(2.167)=0.815 ; \mathrm{p}>.05$ ni en las relaciones con la cultura de acogida $\mathrm{F}(2.019)=2.055 ; \mathrm{p}>.05$. Asimismo, las pruebas corroboran la inexistencia de tal influencia sobre los valores laborales intrínsecos, $\mathrm{F}(0.504)=2.336 ; \mathrm{p}>.05, \mathrm{y}$ 
Inmigración, estrategias de aculturación y valores laborales: un estudio exploratorio

sobre los extrínsecos, $\mathrm{F}(2.229)=1.596$; $\mathrm{p}>$.05. Para reforzar estas pruebas, se realizaron una serie de pruebas de $\mathrm{t}$ de Student a partir de la creación de tres variables ficticias que confirmaron el sentido de los contrastes anteriores.

Posteriormente, se realizó una aproximación categórica al estudio de la cuestión. Con el objetivo de situar a los sujetos en una de las cuatro estrategias de aculturación propuestas por el modelo de Berry se realizó un punto de corte en las variables relativas a las relaciones con las culturas de origen y de acogida tomando como base la mediana de cada dimensión de aculturación (Kosic, 2002; Navas et al, 2004; Berry, 2006b), resultando los siguientes grupos: a) integración $(\mathrm{N}=64$, casos en los que se superaron tanto la mediana de la variable relativa a las relaciones con la cultura de origen y de acogida); b) asimilación ( $\mathrm{N}=45$, sólo se superó la mediana de la variable relativa a las relaciones con la cultura de acogida); c) separación ( $\mathrm{N}=33$, sólo se superó la mediana de la variable relativa a las relaciones con la cultura de origen); y d) marginación $(\mathrm{N}=18$, no se superó la mediana de ninguna de las dos variables). Para conocer la influencia de la estrategia de aculturación adoptada por los sujetos en sus valores laborales, se realizaron pruebas de análisis de varianza o ANOVA, que revelan la existencia de diferencias significativas entre los cuatro grupos resultantes en lo relativo a los valores intrínsecos, $\mathrm{F}(0.276)=98.023$; $\mathrm{p}<.01$, pero no en el caso de los valores extrínsecos, $\mathrm{F}(2.229)=1.156 ; \mathrm{p}>$.05. Para conocer el sentido de tales diferencias en lo referente a los valores laborales intrínsecos, se efectuaron una serie de pruebas post-hoc de Scheffé que revelaron tres grupos, con la existencia de un primer grupo en el cual se sitúan los marginados $(\mathrm{M}=2.61)$ y los separados $(M=2.98)$, un segundo grupo en el que se ubican los asimilados $(\mathrm{M}=3.88)$ y un tercer y último grupo en el cual se posicionan los inmigrantes integrados $(M=4.66)$. Por tanto, se podría decir que marginados y separados tienen unos valores laborales intrínsecos relativamente bajos, los de los inmigrantes asimilados se sitúan en un punto medio y los de los integrados en un punto alto.

Con el objetivo de complementar el análisis realizado y ver cuál de las dos dimensiones de la aculturación tiene mayor peso específico sobre la escala de los valores laborales en la que se han encontrado diferencias significativas entre las distintas estrategias de aculturación, es decir, la subescala de valores intrínsecos, se efectuó una aproximación dimensional a los resultados obtenidos mediante un análisis de regresión múltiple que revela

Tabla 4. Prueba post-hoc de Scheffé: valores intrínsecos en función de la estrategia de aculturación

\begin{tabular}{|c|c|c|c|c|}
\hline & $\mathrm{N}$ & Subconjunto & para alfa & $=0.05$ \\
\hline Marginación & 18 & 2.61 & \multirow{4}{*}{3.88} & \\
\hline Separación & 33 & 2.98 & & \\
\hline$\Lambda$ similación & 45 & & & \\
\hline Integración & 64 & & & 4.66 \\
\hline Sig. & & 0.094 & 1.000 & 1.000 \\
\hline
\end{tabular}


que tanto las relaciones con la cultura de origen como con las de acogida predicen los valores laborales intrínsecos $(\mathrm{F}=143.262 ; \mathrm{p}<.01)$ de los sujetos encuestados. El peso de la influencia sobre los mismos recae mayoritariamente sobre las relaciones con la cultura de acogida y sus miembros $(,=4.674 ; \mathrm{p}<.01)$, mientras que el peso de la cultura propia es menor (,= $1.831 ; \mathrm{p}<.01)$. En total, el modelo explica un $71 \%$ de la varianza de los valores laborales intrínsecos.

\section{DISCUSIÓN Y CONCLUSIONES}

En primer lugar, en base a los resultados obtenidos observamos que la importancia atribuida a los valores laborales extrínsecos supera a la de los intrínsecos. Durante la carrera laboral, los valores extrínsecos tienden a ser más constantes que los intrínsecos (Mortimer, Lorente y Kunda, 1986), sobre todo cuando los individuos no tienen los suficientes recursos económicos para afrontar los retos que plantea la vida diaria. Tan sólo cuando las necesidades materiales están satisfechas, la priorización de los valores extrínsecos decae y la atención de los trabajadores se desplaza hacia otras satisfacciones intrínsecas (Maslow, 1954). Dentro de los aspectos extrínsecos del trabajo, los mejores valorados son el salario, considerado un factor clave en la satisfacción laboral
(Locke, 1976) puesto que brinda al sujeto la posibilidad de acceder a los bienes y servicios, y la estabilidad en el empleo, atributo muy valorado especialmente en periodos de incertidumbre (Topalova y Ruiz Quintanilla, 1992), circunstancia inherente a los procesos migratorios. En cuanto a los valores intrínsecos, observamos que las relaciones con los compañeros es el aspecto que más puntuación obtiene, ya que las relaciones humanas es uno de los elementos que más influye en la satisfacción laboral (Warr, 1987). A cierta distancia se sitúa el deseo de que el puesto de trabajo sea interesante. En cualquier caso, la atribución de determinadas cuotas de importancia a los valores laborales nos corrobora su distribución jerárquica (Ravlin y Megglino, 1987).

Centrándonos en los valores laborales en función de la estrategia de aculturación adoptada por cada individuo, lo primero que destaca es la ausencia de diferencias estadísticamente significativas entre los distintos grupos en lo relativo a los valores laborales extrínsecos, lo que puede explicarse por la Teoría de Necesidades de Maslow (1954). Los distintos elementos que forman parte de los valores laborales extrínsecos se incluirían dentro de las necesidades de déficit y, concretamente, en el seno de aquellas que surgen de la necesidad que tiene la persona de encontrarse segura y protegida (necesidades de

Tabla 5. Regresión: variables predictoras de los valores laborales intrínsecos

\begin{tabular}{|l|c|c|c|l|c|}
\hline & beta & p & t & \multicolumn{2}{|l|}{ Ajuste del modelo } \\
\hline Valores laborales intrínsecos & & & & \multicolumn{2}{|l|}{} \\
\hline Relac.cultura origen & 1.831 & .000 & 6.406 & $\mathrm{R}^{2}$ & .710 \\
Relac.cultura acogida & 4.674 & .000 & 16.573 & $\mathrm{R}^{2}$ corregido & .705 \\
& & & & $\Gamma^{4}$ & 143.262 \\
& & & & Sig. & .000 \\
\hline
\end{tabular}


Inmigración, estrategias de aculturación y valores laborales: un estudio exploratorio

ingresos y recursos, empleo etc.), que ocupan el segundo escalafón de la pirámide, teniendo por debajo tan sólo a las necesidades fisiológicas básicas. La idea básica de la teoría de Maslow estriba en la distribución jerárquica de las necesidades, es decir, las más altas sólo ocupan la atención del sujeto una vez que se han cubierto las necesidades situadas en un plano inferior en la pirámide. Por tanto, los valores extrínsecos resultan básicos para cualquier sujeto, sea cual sea la estrategia de aculturación que adopte, de ahí su preeminencia sobre los intrínsecos, que se distribuyen entre los peldaños superiores de la pirámide, es decir, entre las necesidades de afiliación, reconocimiento y autorrealización.

Situándonos en el plano de los valores intrínsecos, las puntuaciones obtenidas nos confirman la existencia de tres grupos en función de la estrategia de aculturación adoptada. Los inmigrantes que optan por la estrategia de integración son los que más valor otorgan a los aspectos intrínsecos del trabajo. Las personas integradas se benefician tanto de la cultura de origen como de la de la sociedad de acogida y desarrollan una mayor flexibilidad en las relaciones sociales, lo que hace que se logre un mayor equilibrio entre los valores antiguos y los nuevos. Estas personas tienen un mejor ajuste sociocultural y psicológico (Berry et al, 1989; Searle y Ward, 1990; Ward y Kennedy, 1994; Berry 2005, 2006a), lo que supone que, al tener sus necesidades básicas mejor satisfechas, aprecien más los factores intrínsecos del trabajo. El doble apoyo social y el enfoque integracionista favorecen la posesión de un mayor número de valores laborales. Los inmigrantes asimilados tienen menos recursos que aquellos que se integran, puesto que rechazan su cultura propia $y$, de esta manera, pierden una importante fuente de apoyo social e información, la que proviene de las personas de su mismo origen. Estas personas suelen tener más dificultades que los integrados en su proceso de inclusión social (Berry et al, 1989; Berry 2005, 2006a), de ahí que, al ser menor su nivel de satisfacción de necesidades asociadas a los valores extrínsecos, disminuye la importancia que otorgan a los aspectos intrínsecos. La aproximación dimensional al estudio de las relaciones entre las estrategias de aculturación y los valores laborales nos revela que la interacción con la cultura de acogida tiene una influencia notablemente mayor sobre la valoración de los aspectos intrínsecos del trabajo que con la cultura de origen, lo que hace que los inmigrantes separados y marginados, es decir, aquellos que tienen relaciones bajas o inexistentes con la cultura autóctona y con sus miembros, sean los que menos valores intrínsecos tienen. $\mathrm{La}$ identificación con la cultura de acogida y los distintos roles a desempeñar en su seno (entre los que se encuentra el laboral) opera como un factor fundamental a la hora de conseguir una buena adaptación (Bakker, 2005), por lo que los sujetos que optan por las estrategias de separación y marginación tienen más dificultades en el ajuste, lo que hace que no cubran bien sus necesidades básicas y, por tanto, atribuyan más valor a los aspectos extrínsecos en detrimento de los intrínsecos. En este punto cabe traer a colación las tesis de Inglehart (1991, 1997) e Inglehart y Welzel (2006), quienes afirman que en las sociedades industriales avanzadas existe una tendencia global de cambio desde la prioridad por los valores materialistas (extrínsecos) hacia la de valores postmaterialistas (intrínsecos). Esta tendencia, que los autóctonos ya tendrían asumida, se reproduce especialmente entre aquellos 
que mantienen relaciones más fuertes con la cultura de acogida, es decir, integrados y asimilados, por lo que se explicaría su mayor atribución de importancia a los valores intrínsecos.

No obstante, hay que tomar en consideración el hecho de que los valores laborales han sido medidos cuando se ha iniciado ya el proceso de aculturación y no en el momento anterior al asentamiento. Según la Teoría de la disonancia cognitiva de Festinger (1957), la tendencia a evaluar de una forma precisa nuestras opiniones o capacidades puede conducir a que éstas sean modificadas, con el objetivo de disminuir la disonancia cognitiva, ya que existe una fuerza que empuja al individuo a querer reducirla o eliminarla. La disonancia cognitiva crea un desequilibrio interno en el sistema de ideas, creencias, emociones y actitudes, ya que se produce una percepción de incompatibilidad de dos cogniciones simultáneas. Al generarse la disonancia, la persona se ve automáticamente motivada para generar ideas y creencias nuevas para reducir la tensión. Las experiencias de los sujetos pueden también condicionar las actitudes hacia el trabajo, con el fin de minimizar la disonancia cognitiva.

La aculturación es un proceso de cambio cultural que se genera como resultado del contacto continuo de dos o más sistemas culturales diferenciados y que afecta a actitudes, creencias y comportamientos (Berry, 1990, 2005, 2006a). Uno de los principales aspectos de ese proceso adaptativo es el ajuste en el trabajo, en el que los valores laborales de cada individuo juegan un papel capital. Muchos de los resultados del trabajo, tales como la satisfacción laboral o las intenciones de cambio de puesto de trabajo, están afectados por las expectativas anteriores y el ajuste entre los valores laborales del empleado y los que ofrece la organización (Taris, 2003). Por tanto, una vez resaltado el papel relevante de los valores laborales en el proceso de aculturación, a la luz de los resultados podemos concluir diciendo que: a) los valores extrínsecos son más importantes que los intrínsecos para todos los sujetos, ya que son aquellos que conducen a la satisfacción de las necesidades más básicas de los individuos, corroborando así la tesis de la jerarquía de necesidades; b) las personas integradas, aquellas que obtienen el apoyo social de dos fuentes, son las que mayor número de valores laborales tienen y las que más importancia atribuyen a los aspectos intrínsecos del trabajo y; c) las relaciones con la cultura de acogida funcionan como el principal factor determinante de los valores intrínsecos, de ahí que las personas separadas y marginadas tengan un número de valores laborales intrínsecos notablemente menor que las integradas y asimiladas.

\section{REFERENCIAS BIBLIOGRÁFICAS}

Alonso, L.E. (2007). La crisis de la ciudadanía laboral. Barcelona: Anthropos.

Bakker, W. (2005). Emigration and well being: the role of personality and cultural identity in acculturation. Groningen: Rijksuniversiteit.

Berry, J.W., Kim, U., Power, S., Young, M. y Bujaki, M. (1989). Acculturation attitudes inplural societies. Applied Psychology: An International Review, 38,185-206.

Berry, J. W. (1990). Psychology of Acculturation. En: J. Berman (Ed.). Cross 
Inmigración, estrategias de aculturación y valores laborales: un estudio exploratorio

Cultural perspectivas: Nebraska Symposium of Motivation (457-488). Lincoln: University of Nebraska Press.

Berry, J.W. (2005). Acculturation: Living successfully in two cultures. International Journal of Intercultural Relations, 29, 697-712.

Berry, J.W. (2006a). Stress perspectives on acculturation. En D. L. Sam y J. W. Berry (Eds.), The Cambridge handbook of acculturation psychology (43-57). New York: Cambridge University Press.

Berry, J.W. (2006b). Design of acculturation studies. En D. L. Sam y J. W. Berry (Eds.), The Cambridge handbook of acculturation psychology (129-141). New York: Cambridge University Press.

Bronfrenbrener, U. (1987). La ecología del desarrollo humano. Barcelona. Paidós.

Cachón, L. (2003). Itinerarios laborales de los inmigrantes: mercado de trabajo y trayectorias sociales. En A. Tornos (Ed.). Los inmigrantes y el mundo del trabajo, (46-51). Madrid: Universidad Pontificia Comillas

Claes, R. (1987). La centralidad en el trabajo en la vida de los jóvenes. En J.M Peiró, y M.D. Moret (Eds.). Socialización laboral y desempleo juvenil. La transición de la escuela al trabajo, 81-100. Nau Llibres: Valencia.

Dona, G. y Berry, J.W. (1994). Acculturation attitudes and acculturative stress of Central American refugees. International Journal of Psychology, 29 (1), 57 70 .

Falk, R. (2002). La Globalización depredadora: Una crítica. Madrid. Siglo XXI de España Editores.

Festinger, L. (1957). A theory of cognitive dissonance. Standford: Standford University Press.

Herzberg, F.; Maunser, B. y Snyderman, B. B. (1959). The Motivation to Work. New York: John Wiley \& Sons.

Holland, J.L. (1976). Vocational Preferences. En M. Dunnette (Ed.). Handbook of industrial and organizational psychology, (521-570). Chicago: Rand Mc. Nally.

Inglehart, R. (1991). El cambio cultural en las sociedades industriales avanzadas. Madrid: Centro de Investigaciones Sociológicas.

Inglehart, R. (1997). Modernización y posmodernización: el cambio cultural, económico y político en 43 sociedades. Madrid: Centro de Investigaciones Sociológicas.

Inglehart, R. y Welzel, C. (2006). Modernización, cambio cultural y democracia: Modernización, cambio cultural y democracia: la secuencia del desarrollo humano. Madrid. Centro de Investigaciones Sociológicas.

Kosic, A. (1998). Adattamento degli immigranti in relazione al loro bisogno di chiusura cognitive e alle loro strategie di coping. Roma: Universidad de Roma La Sapienza.

Lawler, E. (1973). Motivation in work organizations. Monterrey: Brooks/Cole.

Locke, E.A. (1976). The nature and 
causes of job satisfaction. En I. Ferman y J. Gordus (Eds.). Mental health and the economy, (1297-1349). Chicago: Rand Mc Nally.

Maslow, A.H. (1954). Motivation and personality. New York: Harper.

Martínez Veiga, U. (2004). Trabajadores invisibles: Precariedad, rotación y pobreza de la inmigración en España. Madrid: Catarata.

Mortimer, J. T., Lorence , J. y Kuma, D. S. (1986). Work, family, and personality: transition to adulthood. Norwood. Ablex.

Mow International Research Team. (1987). The Meaning of Working. London. Academic Press.

Navas, M., Pumares, P., Sánchez, J.; García, M. C, Rojas, A., Cuadrado, I., Asensio, M. y Fernández, J. S. (2004). Estrategias de aculturación: la perspectiva de los inmigrantes y de los autóctonos en Almería. Almería: Junta de Andalucía.

Ovejero, A. (2004). Globalización, sociedad y escuela. Cómo hacer frente a los principales problemas actuales desde la psicología social crítica. Valladolid: Servicio de publicaciones Universidad de Valladolid.

Ovejero, A. (2006). Psicología del trabajo en un mundo globalizado: Cómo hacer frente al mobbing y al estrés laboral. Madrid: Biblioteca Nueva.

Ravlin, E.C. y Megglino, B.M. (1987). Issues in work values measurement. Research in Corporate Social Performance and Policy, 9, 153-183.
Rockeach, M. (1973). The Nature of Human Values. San Francisco: Josey Bass.

Topalova, V y Ruiz-Quintanilla, S.A.. (1992). Attitudes Towards Work and the Market Economy in Bulgaria. CHARS Working Paper, 92-36.

Salanova, M., Prieto, F. y Peiró, J.M. (1993). El significado del trabajo: Una revisión de la literatura. En J.M. Peiró, F. Prieto, M.J. Bravo, P. Ripoll, I. Rodríguez, P. Hontangas y M. Salanova (Eds.). Los jóvenes ante el primer empleo: el significado del trabajo y su medida (21-40). Valencia: Nau Llibres.

Salgado, J.F. (2005). Personalidad y deseabilidad social en contextos organizacionales: implicaciones para la práctica y de la psicología del trabajo y de las organizaciones. Papeles del Psicólogo, 92. Disponible en http://www.papelesdelpsicologo.es/vernumero.asp?id=1252.

Searle, W. y Ward, C. (1990). The prediction of psychological and sociocultural adjustment during cross-cultural transitions. International Journal of Intercultural Relations, 14, 449-464.

Shields, M. A. and Ward, M. (2001) Improving nurse retention in the National HealthService in England: the impact of job satisfaction on intention to quit. Journal of Health Economics,20(5), 677-701.

Stitt-Gohdes, W. L. (1997). Career development: Issues of gender, race, and class. Columbus: The Ohio State University.

Taris, R. (2003). Person-Environment fit: A longitudinal study of the interaction between employee characteristics and 
Inmigración, estrategias de aculturación y valores laborales: un estudio exploratorio

work enviromental characteristics. Amsterdam: Vrije Universiteit.

Wahba, M. A. y. Bridwell, L. G (1976). Maslow reconsidered: A review of research on the need hierarchy theory. Organizational Behavior and Human Performance, 15, 212-240.

Ward, C. y Kennedy, A. (1993). Psychological and socio-cultural adjustment during cross-cultural transitions: a comparison of secondary students at home and abroad, International Journal of Psychology, 28, 129-47.

Ward, C. y Kennedy, A. (1994). Acculturation strategies, psychological adjustment, and sociocultural competence during cross-cultural transitions. Internatio- nal Journal of Intercultural Relations, 18, 329-343.

Ward, C. y Kennedy, A. (1999). The measurement of sociocultural adaptation. International Journal of Intercultural Relations, 23(4), 659-677.

Warr, P. (1987). Work, Unemployment and Mental Health. Oxford: Clarendon Press.

WOSY Internal Research Group (1989). Socialización laboral en el joven: un estudio transnacional. Papeles del Psicólogo, 39/40, 32-35.

WOSY International Research Group. (1989b). Interview schedule of the Work Socialization of Youth -Study. Gent: Gent University. 